\title{
Estilos de apego y representaciones maternas durante el embarazo
}

\author{
LUA GRIMALT O. ${ }^{1}$, ELIANA HERESI M. ${ }^{2}$ \\ 1. Psicóloga, Licenciada en Psicología. Universidad Diego Portales. \\ 2. Psicóloga. Magister en Psicología Clínica. Facultad de Psicología. Universidad Diego Portales.
}

\begin{abstract}
Attachment style and maternal representations during pregnancy

Various authors support the theory that the mother-child bond begins in gestation, through a mental representation which the mother constructs during pregnancy. This image would be based on their own childhood experience, which lays the foundation for the transgenerational transmission of the attachment relationship type that will be formed. Objective: To study the relationship between the pregnant mother's history of attachment and her attachment style during pregnancy, including the types of images formed about her future son or daughter as well as herself as a mother. Patients and Method: Three first time pregnant women with different attachment styles were identified through the Parental Bonding Instrument. An in-depth interview was performed with each individual. Flick's adaptation of Grounded Theory was applied during data analysis. Results show that differences exist both in the thematic contents as in the quality, quantity and coherence of their representations depending upon the attachment style. Discussion: These results contribute to the knowledge of the representational world of the pregnant woman, allowing for improved prenatal interventions that will promote secure mother-child attachment.
\end{abstract}

(Key words: Pregnancy, maternal representations, maternal attachment style, mother-child bond).

Rev Chil Pediatr 2012; 83 (3): 239-246

\section{RESUMEN}

De acuerdo a diversos autores, el vínculo madre-hijo se inicia desde la gestación, a través de las representaciones que la madre va construyendo durante el embarazo. Dichas representaciones estarían ancladas en su propia experiencia de apego en la infancia, lo que sentaría las bases de la transmisión transgeneracional de los vínculos de apego. Objetivo: Determinar la relación entre el estilo de apego de la madre y el tipo de representaciones que ésta construye acerca del hijo en gestación, de sí misma como madre y de su propia historia de apego, durante el embarazo. Pacientes y Método: Estudio cualitativo con análisis interpretativo de los datos. Se seleccionaron 3 mujeres primigestas, con distintos estilos vinculares, identificados a través del Parental Bondig Instrument, a las cuales se les realizó una entrevista semi-estructurada, en función de una pauta guía que contenía los principales ejes teóricos del estudio. Para el análisis de los datos, se utilizó la adaptación de Flick de la Grounded Theory. Resultados: Los resultados muestran que existen diferencias tanto en los conte-

Trabajo recibido el 09 de diciembre de 2010, devuelto para corregir el 08 de febrero de 2011, segunda versión 23 de septiembre de 2011, tercera versión el 26 de diciembre de 2011, aceptado para publicación el 04 de febrero de 2012.

Correspondencia a:

Lua Grimalt O.

E-mail: luatomasa@gmail.com. 
nidos temáticos, como en la calidad, cantidad y coherencia de las representaciones maternas, dependiendo del estilo vincular. Conclusión: Los resultados aportan al conocimiento del mundo representacional de la mujer embarazada, lo cual permite el desarrollo de intervenciones prenatales para promover el establecimiento apego seguro madre-hijo.

(Palabras clave: Embarazo, representaciones maternas, estilo de apego materno, vínculo madre-hijo).

Rev Chil Pediatr 2012; 83 (3): 239-246

\section{Introducción}

La importancia de las relaciones de apego para la compresión del origen y mantención de las relaciones cercanas, el desarrollo de la personalidad y la psicopatología, ha sido bien documentada en la literatura ${ }^{1-3}$. El concepto de apego acuñado por Bowlby ${ }^{4}$, da cuenta de un sistema comportamental innato, propio de los seres humanos, que se activa en situaciones de amenaza o aflicción y que tiene la finalidad de recuperar el bienestar, a través de comportamientos destinados a recibir apoyo, cuidado y protección del cuidador primario ${ }^{3}$. El apego conforma un sistema de regulación diádica entre el cuidador y el infante, siendo imprescindible la presencia y disponibilidad del cuidador, ya que dependiendo de las experiencias repetidas (positivas, negativas o ambas), el niño desarrolla una serie de representaciones mentales acerca de la naturaleza de la relación con su figura de apego y acerca de su propia existencia $^{1,4-8}$. Se han descrito estilos o patrones de apego, de acuerdo a los distintos modelos mentales que se van construyendo, las que moldean el procesamiento de la experiencia cognitiva y afectiva de los seres humanos ${ }^{9}$.

El estilo de Apego Seguro describe a sujetos que son capaces de reconocer sus estados de necesidad, pudiendo recurrir en búsqueda de apoyo; su modelo mental internalizado es positivo tanto respecto a si mismo como de los demás. El patrón de apego Inseguro Evitativo, falla en el reconocimiento de la angustia o incomodidad, por tanto, no busca ser consolado; su modelo mental implica una percepción de los otros negativa, en términos de la receptividad y disponibilidad al cuidado, y una percepción también negativa de sí mismo, como alguien que no merece ser cuidado. El patrón de apego Inseguro Ambivalente mostraría un modelo mental caracterizado por la internalización de una relación poco predecible e inestable con sus figuras de apego temprano, manifestando una respuesta emocional hipersensible y expresiones intensificadas de angustia. Así, existiría una tendencia a buscar cuidado y afecto de la figura de apego, al mismo tiempo que sentimientos de temor a ser ignorados o abandonados $^{9-11}$.

En las últimas décadas, se ha reconocido que la relación de apego entre una madre y su hijo se comienza a establecer durante el embarazo, lo cual es conocido como apego prenatal. $\mathrm{Su}$ importancia radica en que existen asociaciones entre el apego prenatal y postnatal ${ }^{12}$, lo que tiene importantes implicancias para el desarrollo cognitivo, emocional y social posterior del bebé, al asociarse directamente con la calidad de la relación madre-hijo y la sensibilidad para captar y responder a las manifestaciones de apego del bebé, luego del nacimiento del mismo ${ }^{4,13-17}$. De este modo, el tipo de representaciones que surjan en la madre, se asociarán posteriormente con su capacidad de regular los estados de estrés del bebé y con la calidad del cuidado y tipo de vinculación que establecerá posteriormente con éste ${ }^{7,18}$.

El inicio de este vínculo prenatal emerge a partir de las representaciones o imágenes internas que la madre va construyendo acerca de su hijo, siendo el embarazo un período clave para la emergencia de dichas representaciones $^{2,19}$. Así también, durante el proceso de convertirse en madre, ocurren importantes procesos en la mujer, en donde su identidad y rol sufren importantes transformaciones. Los modelos que traía sobre sí misma, van adaptándose y reorganizándose en la construcción de un nuevo mundo representacional, en el que se entrelazan modelos sobre su ser mujer, sobre su rol como futura madre, como pareja, 
y de su familia de origen en general ${ }^{20-22}$. En relación a lo anterior, existe evidencia de que el tipo y calidad de las representaciones que la madre genera durante el embarazo, se relacionan estrechamente con su propia historia de apego ${ }^{5}$.

El objetivo de la investigación es establecer la asociación entre el estilo de apego materno y las representaciones que ella construye acerca de sí misma como madre, sobre su futuro hijo y sobre su propia historia de apego, durante el embarazo.

\section{Pacientes y Método}

Se utilizó una metodología cualitativa para producir hallazgos por medio del análisis interpretativo de los datos, con el propósito de descubrir conceptos y relaciones entre éstos y luego organizarlos en un esquema explicativo teórico ${ }^{23}$. El enfoque utilizado se basó en la reformulación del modelo tradicional de Teoría Fundada de Glasser y Strauss ${ }^{24}$, planteada por Uwe Flick ${ }^{25}$.

\section{Participantes y Procedimiento}

La muestra fue definida a priori y se orientó a la búsqueda de 3 mujeres, que fueran casos prototípicos de cada uno de los tres patrones de apego definidos por Ainsworth ${ }^{9}$, que estuvieran cursando entre el cuarto y octavo mes de gestación y que accedieran a participar en el estudio. Se tuvo contacto a la muestra a través de médicos obstetras que accedieron a colaborar con el estudio, los que proporcionaron datos de aquellas pacientes que se consentían a participar y a la vez cumplían con los requisitos de ingreso. De este modo, se procedió a evaluar el estilo de apego a 15 mujeres embarazadas, de modo de encontrar los casos más típicos de los 3 patrones de apego. A los casos seleccionados, se les realizó posteriormente una entrevista semi-estructurada, en base a una pauta guía previamente elaborada, que contenía los principales ejes de la investigación. Todas las participantes firmaron un consentimiento informado, que contenía los aspectos generales de la investigación y los resguardos éticos de la misma.

\section{Instrumentos}

Parental Bonding Instrument (PBI): Mide la percepción de la conducta y actitud de los padres en la infancia y adolescencia, basándose en las dimensiones de cuidado y sobreprotección. Las dos dimensiones forman escalas que se asocian a cinco estilos vinculares parentales: Vinculación Óptima; Vínculo Ausente o Débil; Control con Afecto; Control sin Afecto, Promedio Estadístico ${ }^{26}$. Para efectos de la presente investigación, se consideró como Apego Seguro a la vinculación óptima, como Apego Inseguro Evitativo al Vínculo Ausente o Débil y como Inseguro ambivalente al Control con Afecto y Control sin afecto ${ }^{27,28}$.

Entrevista semiestructurada: La entrevista realizada se caracterizó por la flexibilidad propia de esta técnica de recolección de información, en la que siguiendo una pauta base, se realizaron preguntas abiertas, sosteniendo una conversación fluida, de acuerdo al ritmo de cada entrevistada, que permitieran la expresión de la subjetividad y ahondar en los tópicos generales, previamente reseñados. $\mathrm{La}$ conversación se centró en la exploración de los siguientes ejes temáticos: representaciones del hijo, representaciones de sí misma como mujer y como madre, de la relación de pareja y de la historia relacional con la familia de origen. Las entrevistas fueron grabadas para registrar con mayor fidelidad las narrativas de las madres participantes, luego se realizó una transcripción literal de cada una de ellas.

Análisis de los datos: Se utilizó la Codificación Temática planteada por Uwe Flick ${ }^{25}$, diseñada para comparar grupos o sujetos. El procedimiento implica un proceso de pasos sucesivos, en donde primero se analizaron los casos uno a uno, para posteriormente generar un sistema de categorías, a través del método de la Codificación Abierta. Lo anterior implicó expresar los datos e información surgida de cada entrevista en forma de conceptos, para después agruparlos en categorías mayores, relevantes para la pregunta de investigación. Como resultante del proceso analítico de la codificación abierta, se obtuvo una lista de categorías y sub-categorías. Posteriormente, se cruzaron los casos individuales, en función de las categorías anteriores, con el fin de desarro- 
llar una estructura temática que permitiera la elaboración de similaridades y diferencias entre las representaciones de cada participante y su correspondiente patrón de apego.

\section{Resultados}

\section{Codificación abierta}

En la tabla 1 se exponen las categorías y subcategorías obtenidas a partir del análisis de cada uno de los casos.

\section{Codificación Temática}

\section{2a. Representaciones sobre el hijo}

La gestante con un Vínculo Seguro, manifiesta la existencia de una intensa conexión con su hijo en el período prenatal. Es capaz de sentirse muy unida con éste y a la vez diferenciada, en tanto logra distinguir entre los ritmos de sueño y vigilia propios y los de su hijo. Muestra una gran cantidad y calidad en sus representaciones fetales, siendo estas flexi- bles, específicas y positivas. Describe al futuro hijo a partir de diferentes aspectos de su propia personalidad y en base a movimientos del feto.

La mujer con Vínculo Inseguro Ambivalente, describe al futuro hijo a partir de sus expectativas respecto de éste, teniendo representaciones que surgen a partir de un ideal de persona deseado por ella. Se aprecia dificultad por parte de la madre de generar una representación coherente y específica del hijo. Las representaciones del hijo corresponden a una combinación de las características positivas de sí misma y del padre, presentándose un hijo idealizado, independiente de los movimientos fetales o de la conexión prenatal percibida. Al mismo tiempo, estas representaciones son atravesadas por los temores de la madre, lo que se extiende a los distintos planos representacionales, como miedos en relación al parto, a la personalidad del futuro hijo, a sí misma como madre, relación futura del padre con el hijo, lo que le genera un estado de inseguridad generalizada.

Tabla 1. Codificación abierta

\begin{tabular}{|c|c|c|}
\hline Categoría I & Representaciones sobre el hijo & \\
\hline Sub-categoría 1 & $\begin{array}{l}\text { Características del hijo } \\
\text { Definición: Pensamientos, fantasías y expectativas relativas } \\
\text { al hijo que va a nacer }\end{array}$ & $\begin{array}{l}\text { 1a. Características físicas } \\
\text { 1b. Características psicológicas } \\
\text { 1c. Riqueza y especificidad de las representaciones }\end{array}$ \\
\hline Subcategoría 2 & $\begin{array}{l}\text { Origen de las Representaciones del hijo } \\
\text { Definición: Base sobre la cual se estructuran pensamientos, } \\
\text { fantasías y expectativas del hijo por nacer }\end{array}$ & $\begin{array}{l}\text { 2a. Intensidad y frecuencia de movimientos } \\
\text { 2b. Rasgos de ambos padres } \\
\text { 2c. Rasgos de sí misma }\end{array}$ \\
\hline Categoría II & Representaciones sobre sí misma y de los otros & \\
\hline Sub-categoría 1 & $\begin{array}{l}\text { Representaciones de sí misma como madre } \\
\text { Definición: Ideas, expectativas y creencias acerca de su } \\
\text { futuro rol de madre }\end{array}$ & $\begin{array}{l}\text { 1a. Presencia de representaciones } \\
\text { 1b. Riqueza y especificidad representacional } \\
\text { 1c. Proyección de características propias en el rol } \\
\text { materno }\end{array}$ \\
\hline Subcategoría 2 & $\begin{array}{l}\text { Representaciones de la Pareja como Padre } \\
\text { Definición: Pensamientos y expectativas acerca del rol } \\
\text { paterno }\end{array}$ & $\begin{array}{l}\text { 2a. Representación del rol de padre } \\
\text { 2b. Representación del rol de madre } \\
\text { 2c. Representación de la relación parental }\end{array}$ \\
\hline Sub-categoría 3 & $\begin{array}{l}\text { Matriz de Apoyo } \\
\text { Definición: Sostén que la embarazada busca y recibe de sus } \\
\text { relaciones significativas }\end{array}$ & $\begin{array}{l}\text { 3a. Apoyo de la Madre } \\
\text { 3b. Apoyo de la Pareja } \\
\text { 3c. Apoyo de otros significativos }\end{array}$ \\
\hline Categoría III & Representaciones de la propia historia de apego & \\
\hline Sub-categoría 1 & $\begin{array}{l}\text { Representaciones de su Madre como Madre } \\
\text { Definición: Recuerdos, pensamientos y sentimientos referi- } \\
\text { dos a la figura materna }\end{array}$ & $\begin{array}{l}\text { 3a. Representación general de la madre } \\
\text { 3b. Valoración de la relación con la madre }\end{array}$ \\
\hline Sub-categoría 2 & $\begin{array}{l}\text { Representaciones de sí misma como hija } \\
\text { Definición: Reorganización de los aspectos centrales de la } \\
\text { relación con los padres y posibilidad de diferenciación o } \\
\text { continuidad de ese estilo en la relación con su propio hijo }\end{array}$ & $\begin{array}{l}\text { 2a. Valoración de la relación temprana con la madre } \\
\text { 2b. Diferencias en el estilo de cuidado y protección } \\
\text { recibidos } \\
\text { 2c. Continuidad en el estilo de cuidado y protección }\end{array}$ \\
\hline
\end{tabular}


La mujer con Vínculo Inseguro Evitativo, muestra preocupación por llegar a transmitir estados negativos al hijo durante el embarazo. Se resiste a la emergencia de representaciones del hijo, por su creencia de que la no correspondencia entre el imaginario con la realidad, podría transmitir al bebé la desilusión de no ser como se esperaba. Por otra parte, se esfuerza en tener conductas y actitudes dirigidas a promover el bienestar del hijo (estar calmada, no discutir, evitar situaciones estresantes, etc.), todo esto tomando como base a la creencia de que los estados mentales y emocionales de la madre son transmitidos al feto.

\section{2b. Representaciones sobre sí misma y los otros}

En la madre con Vínculo Seguro, las transformaciones físicas y emocionales propias de la condición de embarazo, llevan a un ajuste paulatino, de nuevas pautas de conducta y a la reacomodación de la rutina. Presenta representaciones de confianza en si misma y en los otros durante el proceso del parto y en la crianza posterior. Su definición del rol de madre lo construye a partir de sus propias características, intereses y actividades, así como también, por las características, intereses y actividades imaginadas del futuro bebé.

La madre con Vínculo Inseguro Ambivalente, presenta una inclinación hacia pensamientos pesimistas en torno al desarrollo positivo del embarazo, razón por la cual, busca estrategias que le proporcionen una matriz de apoyo y seguridad para el cuidado y protección de ella y del futuro hijo. Los cambios emocionales propios de la gestación, son vivenciados de manera intensa y poco autorregulada, observándose una exaltación de los afectos. La seguridad en el parto es prioritariamente puesta en los otros, mientras que se evidencia una falta de confianza en sí misma. Le surgen fantasías aterrorizantes respecto al parto, en relación al dolor físico, como también, por la posibilidad de daño y muerte de ella o del bebé. Los temores relacionados consigo misma como madre, se relacionan con fallar en la protección y cuidado del bebé y con no ser una buena madre.

La mujer con Vínculo Inseguro Evitativo, espera a sentirse segura de sus propias capacidades para ser madre, antes de tomar la decisión de concebir. Posteriormente, trata de evitar tener imágenes o pensamientos acerca de sí misma en el rol de madre, no evidenciando proyecciones respecto a su rol parental futuro. Sin embargo, se representa una forma "buena" de relacionarse con el hijo, en donde ella promueve el apego y la conexión emocional con éste. A diferencia de los otros tipos vinculares, no busca apoyo en amigos o familiares, confiando más en si misma que en los otros, manejando su angustia sin pedir ayuda.

\section{2c. Representaciones acerca de la historia de apego}

En el Vínculo Seguro, se organiza la experiencia de embarazo en relación al apoyo de la figura de apego principal, es decir, la madre. Es a partir de esta base segura, que desarrolla habilidades más eficaces para regular y controlar el estrés. Se aprecia facilidad para recordar el pasado y capacidad de integrar lo vivido en una perspectiva autónoma de sí misma, en un relato coherente y consistente de los recuerdos infantiles, logrando integrar diferentes experiencias en una globalidad. Define las características de sí misma como madre en relación a las características de la propia figura materna, ya sea por semejanzas o diferencias.

En la mujer con Vínculo Inseguro Ambivalente, la madre es descrita como inconsistente en las respuestas de cuidado, debido a lo cual, ella aspira a convertirse en una madre muy diferente, buscando compensar las carencias de cuidado en la infancia y la inseguridad que percibe en la vida adulta. Esta diferenciación, contiene una idealización del propio estilo maternal, proyectándose con altas expectativas respecto a sí misma como madre. Su relato muestra marcada dificultad de articulación de los distintos episodios de la historia vital; no logrando imprimir un sentido global a sus experiencias. Por otro lado, hay una representación de falta de continuidad e inconsistencia en la protección otorgada por los padres, lo que la lleva a tener miedo de no responder a las necesidades de protección del hijo.

Por otra parte, la mujer con Vínculo Inseguro Evitativo, evidencia la intención de cam- 
biar la pauta de crianza materna, basándose en experiencias infantiles negativas. En este caso, la reformulación de los patrones de los padres lleva a definir lo no deseado y a reemplazarlo por algo nuevo. Al mismo tiempo, existe inquietud por revisar los ciclos vividos, dada la importancia de otorgar sentido a la vida. A partir de ello, se aprecia un claro intento de dar significado y reformular la experiencia de rechazo con los propios padres durante el embarazo, obteniendo significados reparadores. En el relato, aparecen detalladamente las experiencias infantiles que no se quieren repetir. Las temáticas son abordadas de modo muy abstracto y generalizado, utilizando muy poco la memoria episódica para enriquecer el relato, el que se presenta con abundantes vacíos.

\section{Discusión}

A partir de los resultados descritos, se pone de manifiesto que durante la gestación del bebé, se desarrollan en la mente de la mujer embarazada imágenes, esperanzas, expectativas, temores y deseos sobre su futuro hijo, como también de sí misma como madre y de su historia de apego ${ }^{19,20,29-31}$. La importancia de estas representaciones radica fundamentalmente en su influencia en la futura conducta interactiva de la madre con el bebé. En el presente estudio, de acuerdo a los distintos tipos vinculares de la muestra, se aprecian diferencias de contenido, calidad y cantidad de las representaciones maternas.

Los resultados obtenidos, que concuerdan con investigaciones precedentes ${ }^{5,16,32}$, señalan que la madre con Apego Seguro presenta una representación equilibrada coherente y flexible del niño, así como una concepción de sí misma como capaz de proveer cuidado y seguridad. La madre con Apego Inseguro Ambivalente representa a su hijo de manera ambigua y a sí misma con dificultades para contenerlo, además de fantasías de muerte y daño. La madre con Apego Inseguro Evitativo se esfuerza por evitar tener representaciones tanto del feto, como de sí misma como futura madre.

Así, en el patrón de apego seguro, se revela un mundo representacional equilibrado tanto de sí misma en el rol materno, como del futuro hijo; también, el relato entrelaza en forma integrada aspectos específicos de su historia de crianza, aportando una visión coherente de sus experiencias con las figuras significativas de la infancia. Se observa continuidad entre el estilo maternal de la propia madre y el estilo proyectado de sí misma en el rol materno, lo que es asumido en forma consciente y reflexiva. La especificidad de las representaciones, sumada a una emocionalidad positiva, puede considerarse como un fuerte precursor de la calidad del vínculo con el futuro infante ${ }^{22,32}$.

En el caso de la gestante con vínculo Inseguro-Ambivalente, las representaciones se presentan cargadas de aprensividad en relación a la posibilidad de muerte, daño o incapacidad de llevar a cabo la función materna. La presencia de pensamientos negativos, inclinados hacia el temor y la pérdida, se extienden a distintos planos representacionales, lo que se relacionaría con la amplificación de los estados internos negativos, de modo que un pequeño malestar se transforma en un peligro de vida, expresando todo con exageración ${ }^{33}$. El pasado es narrado sin un hilo conductor que otorgue sentido al relato; se construye en base a recortes que muestran un cuadro confuso y poco objetivo de la experiencia personal pasada. Dicha confusión en el ámbito de las relaciones al interior de su familia de origen, impide efectuar un proceso de análisis y reflexión objetiva sobre las experiencias del pasado. El estilo maternal transmitido por la propia madre es considerado inconsistente e insegurizador, por lo que la diferenciación con ella es un tema central.

En el caso del estilo vincular Inseguro Evitativo, se presenta un mundo representacional acotado al presente y se evade la generación de representaciones futuras tanto de sí misma como de los otros, incluyendo al hijo, como un intento de evitar una posible decepción en el encuentro de la fantasía y la realidad. El pasado es narrado a partir de conclusiones desarrolladas en el presente, con la finalidad de dar un sentido positivo y de aprendizaje a las experiencias de abandono vivenciadas en la infancia. Se entrega poca información de la historia personal y si lo hace, es a través de la intelectualización, no integrando lo emocional ${ }^{6,11}$. Se evidencia el deseo de diferenciación 
con la propia madre, proyectando aquello positivo que le hubiese gustado recibir de su figura materna. Las personas con este tipo de vínculo tienden a confiar muy poco en lo que sienten y mucho en las explicaciones que se dan, llevando la experiencia a un nivel de abstracción que le da sentido y continuidad al sí mismo ${ }^{33}$.

Dado que las representaciones maternas juegan un papel fundamental en la relación madre-hijo que se comienza a establecer antes del nacimiento del bebé ${ }^{16,34}$ y considerando que el embarazo constituye una situación en la que la mujer se enfrenta a la reestructuración de su mundo representativo, acceder a dichas representaciones permitiría realizar intervenciones preventivas, que apunten a potenciar un apego seguro desde el período prenatal. Esto permitiría interrumpir la transmisión transgeneracional de los patrones de apego inseguros y sus consecuencias en el desarrollo global del niño ${ }^{15,19,35,36}$.

Por último, es importante señalar algunas de las limitaciones del presente estudio. En primer lugar, se considera relevante homogenizar aún más las características de la muestra, de modo de asegurar que las diferencias encontradas no se encuentran relacionadas a factores como el mes de embarazo, la presencia o no de movimientos fetales, la edad de las madres, entre otros, como ha sido señalado en otros estudios $^{16,34}$. En este sentido, sería conveniente que todas las mujeres se encuentren en el mismo período de gestación, como también, respecto al reconocimiento o no de los movimientos fetales, aspecto fundamental en la construcción de representaciones del hijo. Por otra parte, aunque las madres eran todas primigestas, presentaban edades cronológicas diferentes, lo que se evidenció en que las mujeres adultas tenían mayor amplitud de experiencias y un más avanzado proceso de diferenciación, respecto a la madre adolescente. Por último, es relevante realizar estudios que incluyan un mayor tamaño muestral, de modo de corroborar los resultados encontrados.

\section{Referencias}

1.- Lecannelier F: Apego e Intersubjetividad: Influencia de los Vínculos Tempranos en el Desarrollo Humano y la Salud Mental. Santiago: Editorial LOM, 2006: 23-5.
2.- Thompson $R$ : The legacy of early attachments. Child Dev 2000; 71 (1): 145-52.

3.- Hoffer M: Psychobiological Roots of Early Attachment, Current Directions. Psych. Science 2006; 15: 2: 84-8.

4.- Bowlby J: Una Base Segura, Aplicaciones Clínicas de una Teoría del Apego. Barcelona: Editorial Paidós, 1995: 20-39.

5.- Fonagy P: Persistencias Transgeneracionales del Apego: una Nueva Teoría. Revista Aperturas Psicoanalíticas 1999; 3. www. aperturas.org última visita 03-07-2008.

6.- Moneta M: El Apego, Aspectos Clínicos y Psicobiológicos de la Díada Madre-hijo. Santiago: Editorial Cuatro Vientos, 2003: 1-20.

7.- Rozenel V: Los Modelos Operativos Internos (IWM) dentro de la teoría del apego. Revista de Psicoanálisis 2006; 23 www. aperturas.org última visita 09-08-2010.

8.- Sroufe L: Attachment and development: A prospective, longitudinal study from birth to adulthood. Attachment \& Human Development 2003; 7: 349-67.

9.- Ainsworth M: Attachments beyond infancy. American Psychologist 1989; 44: 709-16.

10.- Barthlomew K, Horowitz LM: Attachment Styles Among Young Adults: A Tests Of A Four Category Model. Journal of Personality and Social Psychology 1991; 61: 226-44.

11.- Marrone M: La Teoría del Apego: un Enfoque Actual. Madrid: Psimática, 2001: 103-32.

12.- Muller M: Prenatal And Postnatal Attachment: A Modest Correlation. Journal Of Obstetric, Gynaecologic and Neonatal Nursing 1996; 25: 161-6.

13.- Bakermans-Kranenburg $M$, Juffer $F$, Van Ijzendoorn $M$ : Interventions with video Feedback and Attachment Discussions: Does Type Of Maternal Insecurity Make A Difference? Infant Mental Health Journal 1998; 19 (2): 202-19.

14.- Pajulo M, Helenius H, Mayes L: Prenatal Views Of Baby And Parenthood: Association With Sociodemographic And Pregnancy Factors. Infant Mental Health Journal 2006; 27 (3): 229-50.

15.- Arteaga M: Estudio Comparativo de las Representaciones Maternas durante el Tercer Semestre de Gestación e Incidencia de la Ecografía en su Establecimiento: Embarazos Únicos Normales, Gemelares Normales y Únicos Patológicos. Tesis doctoral Universitat Autónoma de Barcelona. España, 2002: 24-40.

16.- Laxton-Kane M, Slade P: The Rol Of Maternal Prenatal Attachment In A Woman's Experience Of Pregnancy And Implications For The Process Of Care. Journal Of Reproductive And Infant Psychology 2002; 20 (4): 25366. 
17.- Dayton C, Levendosky A, Davidson W, Bogat G: The Child As Held In The Mind Of The Mothers: The Influence Of Prenatal Maternal Representations On Parenting Behaviors. Infant Mental Health Journal 2010; 31 (2): 220-41.

18.- Thun-Hohenstein L, Wienerroither C, Schreuer M, Seim $G$, Wienerroither $H$ : Antenatal Mental Representations About The Child And Mothers-Infant Interaction At Three Month Post Partum. Eur Child adolesc Psyquiatry, 2008; 17: 9-19.

19.- Ammaniti M: Representations and Narratives during Pregnancy. Infant Mental Health Journal 1992; 13: $167-$ 82.

20.- Stern D: La Constelación Maternal. Barcelona: Editorial Paidós, 1997: 209-33.

21.- Hesse E: Discourse, Memory, And The Adult Attachment Interview: A Note With Emphasis On The Emerging Cannot Classify Category. Infant Mental Health Journal 1996; 17: 4-11.

22.- Fonagy P, Steele M, Steele H: Maternal Representations Of Attachment During Pregnancy Predict The Organization Of Infant-Mother Attachment At One Year Of Age. Child Development 1991; 62: 891-905.

23.- Strauss A, Corbin J: Bases De La Investigación Cualitativa. Técnicas y Procedimientos Para Desarrollar La Teoría Fundada. Medellín: Editorial Universidad de Antioquia, 2002: 17-28

24.- Glasser B, Strauss A: The Discovery of Grounded Theory: Qualitative Research. Chicago: Editorial Aldine, 1967: 1-18.

25.- Flick $U$ : Introducción a la Investigación Cualitativa. Madrid: Editorial Morata, 2004: 55-228.

26.- Parker G, Tupling H, Brown L. "A Parental Bonding Instrument", British Journal of Medical Psychology; Londres, The British Psychological Society, 1979; 52: 1-10.
27.- Parker G: The Parental Bonding Instrument: Psychometrics Properties Reviewed. Psychiatric Development 1989; 4: 317-35.

28.- Melis F, Dávila $M$, Ormeño V, Vera V, Greppi C, Gloger $S$ : Estandarización del P.B.I. (Parental Bonding Instrument), versión adaptada a la población entre 16-64 añosdel Gran Santiago. Rev Chil Neuro-Psiquiat 2001; 39 (2): 132-9.

29.- Raphael-Leff J: Pregnancy. The inside story. Londres: Editorial Sheldon Press, 1995: 7-26.

30.- Winnicott D: Los Procesos de Maduración y el Ambiente Facilitador. Buenos Aires: Editorial Paidós, 1993: 29-37.

31.- Ammaniti M: Maternal representations during pregnancy and early infant-mother interactions. Infant Mental Health Journal 1991; 12: 246-55.

32.- Cohen L Slade A: The Psychology and Psychopathology of Pregnancy: Reorganization and Transformation. Handbook of Infant Mental Health. New York, 2002: $22-40$.

33.- Guidano V: El Modelo Cognitivo Postracionalista. Hacia una reconceptualización teórica y crítica. Buenos Aires: Editorial Desclée, 2001: 39-51.

34.- Bielawska-Batorowicz E, Siddiqui A. A Study Of Prenatal Attachment With Swedish And Polish Expectant Mothers. Journal And Reproductive And Infant Psychology 2008; 36 (34): 373-84.

35.- Folliero E: Una Donna Diventa Madre, Una Madre Resta Donna: Uno Studio Empirico Sulla Relazione Tra Rappresentazioni Materne In Gravidanza E Funzione Riessiva. Universita Católica del Sacro Cuore di Milano. Italia, 2003: 79-80.

36.- Taskin E, Okman G: Maternal Representations during Pregnancy and Early Motherhood. Infant Mental Health Journal 2004; 25 (1): 16-27. 